\title{
Another look at Bayes map iterated filtering
}

\author{
Dao Nguyen ${ }^{1}$ \\ Departament of Statistics, University of Michigan, Ann Arbor, MI 48109, Michigan, USA
}

\begin{abstract}
A new proof of convergence of Bayes map iterated filtering algorithm, based on supermartingale inequality, is proposed. Relying on log concavity assumption, this approach with verifiable conditions is simpler than the previous approach and is generalizable to more sophisticated algorithms. The assumption could be relaxed at the cost of more technical details.
\end{abstract}

Keywords: Iterated filtering, Bayes map, Hidden Markov model, Stochastic approximation, Time series

\section{Introduction}

Inference methodology for stochastic dynamic systems is often termed "plug-and-play" if only simulated estimation is needed to plug into the inference procedure (Bretó et al., 2009). The plug-and-play methodology has been used to free researchers from demanding closed-form expression requirements for transition probabilities, imposed by previously available statistical methodology, allowing researchers to broaden classes of modeling and considering novel hypotheses. Unlike the mainstream statistical techniques (Expectation-Maximization algorithms and Bayesian Markov Chain Monte Carlo), plug-and-play approaches are a relatively recent and exciting development because of their less restrictive requirements. Examples of plug-and-play methodologies follow the frequentist paradigm (Ionides et al., 2011; Lindström et al., 2012), the Bayesian paradigm (Andrieu et al., 2010; Toni et al., 2009), or work by matching selected summary statistics (Wood, 2010). Amongst these approaches, we are interested in iterated filtering because of its computational efficiency. Iterated filtering was originally proposed by Ionides et al. (2006) and later theoretically developed by Ionides et al. (2011). Recently, Lindström et al. (2012) extended it to improve on numerical performance while Doucet et al. (2013) expanded it to include filtering/smoothing with quite attractive theoretical properties. Ionides et al. (2015) generalized Lindström et al. (2012)'s approach and combine the idea with data cloning (Lele et al., 2007), developed a Bayes map iterated filtering with an entirely different theoretical approach. We revisit the approach of Ionides et al. (2015) using a supermartingale inequality, which relies on easily verifiable conditions, thus of more general interests. The paper is organized as follows. In the next section, we introduce some notations and develop the framework of Bayes map iterated filtering. In Sections 3, we prove the convergence of this approximation filter to the maximum likelihood estimator (MLE) by super-martingale inequality.

\section{Problem definition}

We consider a general latent variable model $(X, Y, \theta)=\left(X_{1: n}, Y_{1: n}, \theta\right)$ for $n \in N$ where $X_{i: j}$ denotes $\left(X_{i}, \ldots, X_{j}\right)$ and $(X, Y)$ is a discrete time Markov chain with $X$ is also a Markov chain, taking values in some measurable space $\mathscr{X}$. We suppose that $X$ is unobserved while $Y$ is observed and taking fixed value $y$ in observation space $\mathscr{Y}$. We also suppose that the joint density $f_{X Y}(x, y ; \theta)$ of a random variables $(X, Y)$ depending on a parameter $\theta \in \Theta$. The marginal densities of $X$ and $Y$ are denoted $f_{X}(x ; \theta)$ and $f_{Y}(y ; \theta)$ respectively while the conditional density of the observed variable $Y$ given the latent variable $X$, also known as measurement model, is denoted as $f_{Y \mid X}(y \mid x ; \theta)$. Let $f$ be a Markov kernel acting on

Email address: nguyenxd@umich.edu (Dao Nguyen)

${ }^{1}$ Tel. +17347095738; Fax:+17347634676 
$\mathscr{X}$ to itself, $g$ be a Markov kernel acting from $\mathscr{X}$ to $\mathscr{Y}$. The likelihood function $L(\theta)=f_{Y}(y ; \theta)=\int f_{\theta}(y \mid x) g_{\theta}(x) d x$. It is assumed that the likelihood is intractable. We are interested in computing the MLE. Set $\ell(\theta)=\log L(\theta)$, and

$$
\theta_{\star}=\arg \max _{\theta \in \Theta} \ell(\theta)
$$

The data cloning approach to computing $\theta_{\star}$ is to introduce the set of posterior distributions $\left\{\pi_{n}, n \geq 1\right\}$, where

$$
\pi_{n}(\theta \mid y)=\frac{e^{n \ell(\theta)} \pi(\theta)}{\int e^{n \ell(\theta)} \pi(\theta) d \theta},
$$

for some prior distribution $\pi$. Lele et al. (2007) show that if $\Theta_{n} \sim \pi_{n}$, then $\Theta_{n}$ converges in probability to $\theta_{\star}$, and $\sqrt{n}\left(\Theta_{n}-\theta_{\star}\right)$ converges weakly to a Gaussian distribution. The precise statement of their result is as follows.

Assumption 1. There exist $\theta_{\star}$ such that $\theta \mapsto \ell(\theta)$ attains a local maximum at $\theta_{\star}$.

Assumption 2. $\pi$ is continuous at $\theta_{\star}, \theta \mapsto \ell(\theta)$ is of class $C^{2}$ in a neighborhood of $\theta_{\star}$ and $-H_{\theta_{\star}}$ is positive definite, where $\left.H_{\theta_{\star}} \stackrel{\text { def }}{=} \nabla^{2} \ell(\theta)\right|_{\theta=\theta_{\star}}$.

Assumption 3. For any $\delta>0, \gamma(\delta)<\ell\left(\theta_{\star}\right)$, where $\gamma(\delta) \stackrel{\text { def }}{=} \sup \left\{\ell(\theta),\left\|\theta-\theta_{\star}\right\|>\delta\right\}$.

Theorem 4. Lele et al. (2007) Assume Assumptions 1 - 3. Set $\Sigma \stackrel{\text { def }}{=}\left\{-H_{\theta_{\star}}\right\}^{-1 / 2}$, and $\Psi_{n} \stackrel{\text { def }}{=} \sqrt{n} \Sigma\left(\Theta_{n}-\theta_{\star}\right)$. As $n \rightarrow \infty, \Theta_{n}$ converges in probability to $\theta_{\star}$, and $\Psi_{n}$ converges weakly to $N\left(0, I_{d}\right)$.

The main ingredient of the proof is the following Taylor expansion. For any $u \in \mathbb{R}^{p}$, there exists $t \in(0,1)$ such that

$$
\ell\left(\theta_{\star}+\Sigma u\right)-\ell\left(\theta_{\star}\right)=-\frac{1}{2}\|u\|^{2}+\frac{1}{2} u^{\prime} \Sigma\left(\nabla^{2} \ell\left(\theta_{\star}+t \Sigma u\right)-\nabla^{2} \ell\left(\theta_{\star}\right)\right) \Sigma u .
$$

As a consequence, we get the following lemma.

Lemma 5. Assume Assumption 1 - 2. Then for all $\theta \in \Theta$,

$$
\lim _{n \rightarrow \infty} n\left(\ell\left(\theta_{\star}+\frac{1}{\sqrt{n}} \Sigma \theta\right)-\ell\left(\theta_{\star}\right)\right)=-\|\theta\|^{2} / 2
$$

and the convergence is uniform on compact sets.

One limitation of the data cloning approach is that it is not clear how the technique can be used in situations where $f_{\theta}$, the density of the state process is intractable. We extend the data cloning approach by iterating sequentially the filtering of the time series data.

\section{Main result}

Suppose we wish to solve the maximization problem

$$
\theta_{\star} \stackrel{\text { def }}{=} \arg \max _{\theta \subset \mathbb{R}^{\mathrm{d}}} \ell(\theta)
$$

for some smooth function $\ell$, that we think of as a log-likelihood function. Our motivation comes from partially observed Markov processes, and latent variables models where $\ell$ can be written as $\ell(\theta) \stackrel{\operatorname{def}}{=} \log \int q_{\theta}(x) f_{\theta}(y \mid x) d_{X_{0}}$. But for the time being $\ell$ is arbitrary.

Let us write $\mathcal{P}$ to denote the space of all probability measures on $\mathbb{R}^{d}$. Let $K_{n}$ denote the probability kernel on $\mathbb{R}^{d} \times \mathcal{B}\left(\mathbb{R}^{d}\right)$ given by

$$
K_{n}(\theta, d \theta)=\frac{1}{\sigma_{n}^{d}} \mathrm{~K}\left(\sigma_{n}^{-1}(\vartheta-\theta)\right) d \vartheta
$$


for positive scale parameter $\sigma_{n}$, and where $K$ is the density of the normal distribution on $\mathbb{R}^{d}$ with mean zero and covariance $\Lambda$. Without any loss of generality in the theory we assume that $\Lambda=I_{d}$, the $d \times d$ identity matrix.

We consider this Bayesian operator $\mathrm{B}_{n}: \mathcal{P} \rightarrow \mathcal{P}$ which transforms the probability measure $\mu$ into $B_{n}(\mu)$ defined as

$$
\begin{gathered}
\mathrm{B}_{n}(\mu)(A)=\frac{\int \mu(d \theta) \int K_{n}(\theta, d \vartheta) e^{\ell(\vartheta)} 1_{A}(\vartheta)}{\int \mu(d \theta) \int K_{n}(\theta, d \vartheta) e^{\ell(\vartheta)}} \\
=\frac{\int \mu(d \theta) \int \mathrm{K}(d u) e^{\bar{\ell}\left(\theta+\sigma_{n} u\right)} 1_{A}\left(\theta+\sigma_{n} u\right)}{\int \mu(d \theta) \int \mathrm{K}(d u) e^{\bar{\ell}\left(\theta+\sigma_{n} u\right)}},
\end{gathered}
$$

by the change of variable $\vartheta=\theta+\sigma_{n} u$, where $\bar{\ell}=\ell-\ell\left(\theta_{\star}\right)$. Given some initial probability measure $\pi_{0} \in \mathcal{P}$ we consider the sequence of probability measures $\left\{\pi_{n}, n \geq 0\right\}$, defined recursively by

$$
\pi_{n}=\mathrm{B}_{n}\left(\pi_{n-1}\right), n \geq 1 \text {. }
$$

The goal here is to show that as $n \rightarrow \infty, \pi_{n}$ concentrates around the solution $\theta_{\star}$ of the maximization problem (1). We assume that $\ell$ is strongly concave.

Assumption 6. The function $\ell: \mathbb{R}^{d} \rightarrow \mathbb{R}$ is concave, admits a unique maximum $\theta_{\star} \in \mathbb{R}^{d}$, and is twice differentiable. Furthermore, there exists $0<\kappa \leq L<\infty$ such that for all $\theta \in \mathbb{R}^{d}$, the spectrum of $\nabla l^{(2)}(\theta)$ is contained in $[-L,-\kappa]$

Assumption 7. Assume $\sum \sigma_{n}^{2}<\infty$.

By standard first-order optimality conditions, $\nabla \ell\left(\theta_{\star}\right)=0$. Assumption 6 implies that for all $\theta, u \in \mathbb{R}^{d}$

$$
-\kappa\|u\|^{2} \geq \nabla^{(2)} \ell(\theta) \cdot(u, u) \geq-L\|u\|^{2} .
$$

We obtain the following lemma.

Lemma 8. Suppose Assumption 6, the following holds,

$$
\pi_{n}(h)=\frac{\int \pi_{n-1}(d \theta) e^{\ell(\theta)} h(\theta)}{\int \pi_{n-1}(d \theta) e^{\ell(\theta)}}+\sigma_{n}^{2} \epsilon_{n, 3},
$$

for some $\epsilon_{n, 3}<\infty$.

Proof. See Supplement S1.

Lemma 9. Denote

$$
\pi_{N}^{1}(h)=\frac{\int \pi_{N}(d \theta) e^{\ell(\theta)} h(\theta)}{\int \pi_{N}(d \theta) e^{\ell(\theta)}},
$$

and for an integer $m>1$,

$$
\pi_{N}^{m}(h)=\frac{\int \pi_{N}^{m-1}(d \theta) e^{\ell(\theta)} h(\theta)}{\int \pi_{N}^{m-1}(d \theta) e^{\ell(\theta)}},
$$

then

$$
\pi_{N}^{k}(h)=\pi_{N+k}(h)-\sum_{i=N+1}^{N+k} \epsilon_{i, 3} \sigma_{i}^{2},
$$

for some bounded sequence $\left\{\epsilon_{i, 3}, i \geq 1\right\}$.

Proof. See Supplement S2. 
Theorem 10. Theorem 1 of (Robbins and Siegmund, 1985). Let $(\Omega, \mathrm{F}, \mathrm{P})$ be a probability space and $\mathrm{F}_{1} \subset \mathrm{F}_{2} \subset \ldots$, a sequence of sub $-\sigma$-algebras of $F$. For each $n=1,2, \ldots$, let $z_{n}, \beta_{n}, \xi_{n}$ and $\zeta_{n}$ be non-negative $\mathrm{F}_{\mathrm{n}}$-measurable random variable such that

$$
\mathrm{E}\left(\mathrm{z}_{\mathrm{n}+1} \mid \mathrm{F}_{\mathrm{n}}\right) \leq \mathrm{z}_{\mathrm{n}}\left(1+\beta_{\mathrm{n}}\right)+\xi_{\mathrm{n}}-\zeta_{\mathrm{n}}
$$

$\lim _{\mathrm{n} \rightarrow \infty} \mathrm{z}_{\mathrm{n}}$ exists and is finite and $\sum_{1}^{\infty} \zeta_{n}<\infty$ a.s. on

$$
\left\{\sum_{1}^{\infty} \beta_{\mathrm{n}}<\infty, \sum_{1}^{\infty} \xi_{\mathrm{n}}<\infty\right\} .
$$

Theorem 11. Suppose that Assumptions 6 and 7 hold. Suppose also that $\pi_{0}$ is absolutely continuous w.r.t. the Lebesgue measure and $\breve{\Theta}_{n} \sim \pi_{n}$. Then $\breve{\Theta}_{n}$ converges a.s to $\theta_{*}$ as $n \rightarrow \infty$.

Proof. By Lemma 8,

$$
\begin{gathered}
\pi_{n}(h)=\frac{\int \pi_{n-1}(d \theta) e^{\ell(\theta)} h(\theta)}{\int \pi_{n-1}(d \theta) e^{\ell(\theta)}}+\sigma_{n}^{2} \epsilon_{n} \\
=\pi_{n-1}(h)+\left\{\frac{\int \pi_{n-1}(d \theta) e^{\bar{\ell}(\theta)} h(\theta)}{\int \pi_{n-1}(d \theta) e^{\bar{\ell}(\theta)}}-\pi_{n-1}(h)\right\}+\sigma_{n}^{2} \epsilon_{n},
\end{gathered}
$$

where $\bar{\ell}(\theta)=\ell(\theta)-\ell\left(\theta_{\star}\right)$, and $\left\{\epsilon_{n}\right\}$ is a bounded sequence. For $t \in[0,1]$, we define $\pi_{n, t}(d \theta)=\frac{1}{c_{n, t}} \pi_{n}(d \theta) e^{t \bar{\ell}(\theta)}$, where $c_{n, t}=\int \pi_{n}(d \theta) e^{\bar{t}(\theta)}$. We have

$$
\begin{aligned}
\frac{\int \pi_{n-1}(d \theta) e^{\bar{\ell}(\theta)} h(\theta)}{\int \pi_{n-1}(d \theta) e^{\bar{\ell}(\theta)}} & -\pi_{n-1}(h)=\int \pi_{n-1,1}(d \theta) h(\theta)-\int \pi_{n-1,0}(d \theta) h(\theta) \\
& =\int_{0}^{1}\left\{\frac{d}{d t} \int \pi_{n-1, t}(d \theta) h(\theta)\right\} d t
\end{aligned}
$$

where $h(\theta)=\ell\left(\theta_{\star}\right)-\ell(\theta)$

$$
\begin{gathered}
=\int_{0}^{1}\left\{\int \frac{d}{d t}\left[\frac{1}{c_{n-1, t}} \pi_{n-1}(d \theta) e^{t \bar{\ell}(\theta)}\right] h(\theta)\right\} d t \\
=\underbrace{\int_{0}^{1}\left\{\int\left[\frac{d}{d t} \frac{1}{c_{n-1, t}}\right] \pi_{n-1}(d \theta) e^{t \bar{\ell}(\theta)} h(\theta)\right\} d t}_{A}+\underbrace{\int_{0}^{1}\left\{\int \frac{1}{c_{n-1, t}} \pi_{n-1}(d \theta) \bar{\ell}(\theta) e^{t \bar{\ell}(\theta)} h(\theta)\right\} d t}_{B} .
\end{gathered}
$$

Since

we have

$$
\frac{d}{d t} \frac{1}{c_{n-1, t}}=\frac{-\frac{d}{d t} c_{n-1, t}}{c_{n-1, t}^{2}}=\frac{-\int \bar{\ell}(\tilde{\theta}) \pi_{n-1}(d \tilde{\theta}) e^{t \bar{\ell}(\tilde{\theta})}}{c_{n-1, t}^{2}}
$$

$$
\begin{gathered}
A=\int_{0}^{1}-\left\{\int \frac{\bar{\ell}(\tilde{\theta}) \pi_{n-1}(d \tilde{\theta}) e^{t \bar{\ell}(\tilde{\theta})}}{c_{n-1, t}} \int \frac{\pi_{n-1}(d \theta) e^{t \bar{\ell}(\theta)} h(\theta)}{c_{n-1, t}}\right\} d t \\
=\int_{0}^{1}-E[\bar{\ell}(\tilde{\Theta})] E[h(\tilde{\Theta})] d t,
\end{gathered}
$$

and

$$
B=\int_{0}^{1} E[\bar{\ell}(\tilde{\Theta}) h(\tilde{\Theta})] d t,
$$


where $\tilde{\Theta}$ is a random variable follows the distribution $\pi_{n-1, t}$ as defined above. So

$$
\begin{aligned}
& \int_{0}^{1}\left\{\frac{d}{d t} \int \pi_{n-1, t}(d \theta) h(\theta)\right\} d t \\
= & \int_{0}^{1} \operatorname{Cov}_{n-1, t}(\bar{\ell}(\tilde{\Theta}), h(\tilde{\Theta})) d t,
\end{aligned}
$$

where the covariance and variance are w.r.t to the distribution $\pi_{n-1, t}$. Since we choose $h(u)=\ell\left(\theta_{\star}\right)-\ell(u), u \in \Theta$, we have

$$
\pi_{n}(h)=\pi_{n-1}(h)-\int_{0}^{1} \operatorname{Var}_{n-1, t}(\bar{\ell}(\tilde{\Theta})) d t+\sigma_{n}^{2} \epsilon_{n} .
$$

We then make use of the super-martingale theorem (Theorem 10) to conclude that $\pi_{n}(h)$ converges to a finite limit $\pi_{u}(h)$ and $\sum_{n} \int_{0}^{1} \operatorname{Var}_{n-1, t}(\bar{\ell}(\tilde{\Theta})) d t<\infty$. Given $\epsilon$, there exists an $N$ such that $\left|\pi_{N}(h)-\pi_{u}(h)\right|<\epsilon$. By Lemma 8 ,

$$
\pi_{N+1}(h)=\frac{\int \pi_{N}(d \theta) e^{\ell(\theta)} h(\theta)}{\int \pi_{N}(d \theta) e^{\ell(\theta)}}+\sigma_{N+1}^{2} \epsilon_{N+1},
$$

SO

$$
\pi_{N}^{m}(h)=\frac{\int \pi_{N}(d \theta) e^{n \ell(\theta)} h(\theta)}{\int \pi_{N}(d \theta) e^{n \ell(\theta)}}=\pi_{N+m}(h)+\sum_{i=N+1}^{N+m} \epsilon_{i, 4} \sigma_{i}^{2} .
$$

Since $\pi_{N}^{m}(h)$ is $m$ iteration of data cloning of $\pi_{N}(h)$, by Theorem $4, \pi_{N}^{m}(h)$ converges to $\delta_{\theta_{\star}}$ as $m \rightarrow \infty$. As above $\sum_{i=N+1}^{N+m} \epsilon_{i, 4} \sigma_{i}^{2}$ and $\epsilon$ can be made arbitrarily small, hence $\pi_{u}(h)$ converges to $h\left(\theta_{\star}\right)=0$. Thus, we can conclude that $\mathrm{E}\left(\bar{\ell}\left(\breve{\Theta}_{n}\right)\right) \rightarrow 0$ or $\mathrm{E}\left(\ell\left(\theta_{\star}\right)-\ell\left(\breve{\Theta}_{n}\right)\right) \rightarrow 0$. Since $\ell\left(\theta_{\star}\right)-\ell\left(\breve{\Theta}_{n}\right) \geq 0$, we have $\ell\left(\theta_{\star}\right)-\ell\left(\breve{\Theta}_{n}\right) \rightarrow 0$. From Assumption 6, $\ell$ admits a unique maximum $\theta_{\star}$ and is twice differentiable, which implies that $\breve{\Theta}_{n} \rightarrow \theta_{\star}$.

\section{Acknowledgements}

This research was funded in part by National Science Foundation grant DMS-1308919 and National Institutes of Health grants 1-U54-GM111274 and 1-U01-GM110712.

\section{References}

Andrieu, C., Doucet, A., Holenstein, R., 2010. Particle Markov chain Monte Carlo methods. Journal of the Royal Statistical Society: Series B (Statistical Methodology) 72 (3), 269-342.

Bretó, C., He, D., Ionides, E. L., King, A. A., 2009. Time series analysis via mechanistic models. Annals of Applied Statistics 3, $319-348$.

Doucet, A., Jacob, P. E., Rubenthaler, S., 2013. Derivative-free estimation of the score vector and observed information matrix with application to state-space models. ArXiv:1304.5768.

Ionides, E. L., Bhadra, A., Atchadé, Y., King, A., 2011. Iterated filtering. Annals of Statistics 39, 1776-1802.

Ionides, E. L., Bretó, C., King, A. A., 2006. Inference for nonlinear dynamical systems. Proceedings of the National Academy of Sciences of the USA 103, 18438-18443.

Ionides, E. L., Nguyen, D., Atchadé, Y., Stoev, S., King, A. A., 2015. Inference for dynamic and latent variable models via iterated, perturbed Bayes maps. Proceedings of the National Academy of Sciences of the USA 112 (3), 719-724.

Lele, S. R., Dennis, B., Lutscher, F., 2007. Data cloning: Easy maximum likelihood estimation for complex ecological models using Bayesian Markov chain Monte Carlo methods. Ecology Letters 10 (7), 551-563.

URL http://dx.doi.org/10.1111/j.1461-0248.2007.01047.x

Lindström, E., Ionides, E. L., Frydendall, J., Madsen, H., 2012. Efficient iterated filtering. In: 16th IFAC Symposium on System Identification.

Robbins, H., Siegmund, D., 1985. A convergence theorem for non negative almost supermartingales and some applications. In: Herbert Robbins Selected Papers. Springer, pp. 111-135.

Toni, T., Welch, D., Strelkowa, N., Ipsen, A., Stumpf, M. P., 2009. Approximate Bayesian computation scheme for parameter inference and model selection in dynamical systems. Journal of the Royal Society Interface 6, 187-202.

Wood, S. N., 2010. Statistical inference for noisy nonlinear ecological dynamic systems. Nature 466 (7310), $1102-1104$. 